\title{
Féeries
}

Études sur le conte merveilleux, XVII ${ }^{\mathrm{e}} \mathrm{XIX} \mathrm{X}^{\mathrm{e}}$ siècle

1 | 2004

Le Recueil

\section{Les premiers recueils de contes de fées}

\section{Anne Defrance}

\section{OpenEdition}

\section{Journals}

Édition électronique

URL : http://journals.openedition.org/feeries/66

DOI : $10.4000 /$ feeries.66

ISSN : 1957-7753

\section{Éditeur}

UGA Éditions/Université Grenoble Alpes

\section{Édition imprimée}

Date de publication : 1 février 2004

Pagination : 27-48

ISSN : 1766-2842

\section{Référence électronique}

Anne Defrance, «Les premiers recueils de contes de fées ». Féeries [En ligne], 1 | 2004, mis en ligne le 29 mars 2007, consulté le 08 septembre 2020. URL : http://journals.openedition.org/feeries/66 ; DOI : https://doi.org/10.4000/feeries.66

Ce document a été généré automatiquement le 8 septembre 2020.

(c) Féeries 


\title{
Les premiers recueils de contes de fées
}

\author{
Anne Defrance
}

ON N'AURA CERTES PAS ATTENDU la naissance du conte de fées littéraire, à la fin du XVII siècle, pour voir fleurir les recueils. Cette pratique éditoriale a, auparavant, profité à bien des genres et à bien des éditeurs. Les recueils littéraires, essentiellement poétiques dans la première moitié du XVII ${ }^{\mathrm{e}}$ siècle, se multiplient et se diversifient dans la seconde, proposant aux lecteurs des florilèges de pièces souvent trop brèves pour être publiées seules. Les ana naissent au milieu du siècle, offrant leurs mélanges de bons mots et d'anecdotes, de pensées détachées. Une infralittérature de consommation facile et rapide rencontre un public, qui peut réinvestir en société le fruit d'un glanage ordonné ou non, selon son bon plaisir. Des recueils à prétention davantage littéraire au même moment prolifèrent, mêlant morceaux en prose ou en vers tels que lettres, poèmes, discours ; pièces nouvelles et rééditions s'y côtoient. À partir de 1672, Le Mercure galant, fondé par Donneau de Visé ${ }^{1}$, offre ses chroniques des événements du grand monde et de la Cour et ses informations sur les publications contemporaines. Pour les agrémenter, des nouvelles y sont intercalées et même, dans la dernière décennie, quelques contes de fées, puisqu'ils sont à la mode. Parallèlement, la pratique éditoriale $\mathrm{du}$ recueil est combattue par les critiques, qui dénoncent l'aisance avec laquelle les éditeurs fabriquent du neuf avec de l'ancien, en maquillant au besoin la publication d'un titre nouveau. En 1699, l'abbé Pierre de Villiers, dans ses Entretiens sur les contes de fées, et sur quelques autres ouvrages du tems, fustige non seulement les contes de fées, mais encore ces nombreux "recueils» auxquels il consacre tout un chapitre. Il s'insurge notamment contre les ana, qui prétendent avoir recueilli des propos ou des textes trop vite attribués à des auteurs célèbres, et reproche globalement aux recueils de relever d'une démarche mensongère, abusive, aux fins purement commerciales. Le terme, à l'époque, peut facilement glisser vers le négatif : le Dictionnaire de l'Académie, qui se présente pourtant lui-même comme un " recueil $^{2}$ ", en donne cette définition : " ramas, assemblage de diverses actes, écrits, et autres sortes de pièces ». Que l'on se reporte à l'entrée « ramas »: " assemblage de diverses choses. Il a fait un ramas de toutes sortes de méchans livres, de curiosités. [...] Il n'a guère d'usage qu'en parlant de l'amas, de 
l'assemblage de choses qu'on regarde comme étant de peu de considération.» Quand Villiers se plaint de "ces recueils, [...] petites historiettes, et enfin de ce ramas de contes de fées, qui nous assassinent depuis un an ou deux ", il met au rebut tout ensemble une forme-réceptacle et son contenu, les petits genres, dont il attribue l'existence à des « ignorans entêtés de l'envie de faire des livres ${ }^{3}$ ».

2 Le dictionnaire de l'Académie donne au verbe « recueillir » plusieurs sens : il s'agit soit de « rassembler, ramasser plusieurs choses dispersées », et d'abord les fruits de la terre, le verbe « récolter » n'existant pas encore en cette fin de siècle ${ }^{4}$, soit de « compiler, réunir dans un corps plusieurs choses de même nature éparses dans un auteur, dans plusieurs auteurs ». Ces deux acceptions rendent compte de la malléabilité de la forme-recueil, de sa variabilité. Elle rend possible aussi bien le regroupement de textes d'un même auteur ou de plusieurs, d'un même genre ou de genres variés, et le croisement de ces différentes possibilités augmente encore leur diversité.

3 C'est de cette matrice plurielle et composite que naquirent les premiers contes de fées. Quels sont les rapports de ce genre nouveau à la forme disposée à les promouvoir ? Quels en ont été les types de prédilection, les apports respectifs du conte et du recueil quant à une pratique éditoriale? On s'appuiera sur des exemples empruntés aux productions inaugurales pour répondre à ces questions.

Le conte-recueil

4 Les contes de fées sont souvent présentés par les premiers conteurs de la fin du XVII siècle soit comme des récits recueillis à la bouche des mies, soit comme les produits d'un récolement de documents anciens, dans lesquels la matière merveilleuse dormait en silence. D'une certaine manière, le conte est donc en soi recueil à part entière, fruit d'une "cueillette", d'un rassemblement et d'une compilation, sinon des fruits de la terre, au moins de ce qui s'ancre dans un terroir, relie un peuple à ses origines.

5 En ce qui concerne la technique du récit, les conteurs de féeries aiment souvent à exhiber la trace de cette oralité primitive. Leurs narrateurs pointent ici ou là quelques zones d'ombre, qui ramènent à la source confuse, lointaine, de cette parole rapportée, transmise, dont l'origine, souvent non mentionnée, se perd dans les brouillards du temps et l'estompe de la mémoire.

6 L'actualisation du conte passe par une inscription dans un double espace, individuel et collectif, diachronique et synchronique. En 1695, l'une des toutes premières conteuses, $\mathrm{M}^{\text {lle }}$ Lhéritier, nièce de Perrault, plusieurs fois récompensée pour ses œuvres poétiques, offre à $\mathrm{M}^{\mathrm{me}}$ de Murat L'Adroite Princesse ou les Aventures de Finette, nouvelle. Le don de ce conte, qu'elle appelle aussi son "historiette", relève d'une pratique de l'échange interpersonnel entre femmes de lettres (sa dédicataire n'a pas encore publié de contes de fées, mais dans son exil à Loches, elle et ses amis s'en racontent ${ }^{5}$ ):

Vous faites les plus jolies Nouvelles du monde en Vers; mais en Vers aussi doux que naturels : je voudrois bien, charmante Comtesse, vous en dire une à mon tour [...] je ne voudrois ni Vers ni Prose pour vous la conter : point de brillans, point de rimes; un tour naïf m'accommode mieux; en un mot, un recit sans façon \& comme on parle : je ne cherche que quelque moralité6.

7 Cet échange duel est ensuite, plus largement, recadré par $\mathrm{M}^{\text {lle }}$ Lhéritier dans une pratique collective, celle d'un jeu de salons : elle rappelle le goût contemporain pour les proverbes : "C'est la mode ; vous les aimez: je m'accommode à l'usage avec plaisir ». Une fois l'axe diachronique de sa communication posé, elle s'inscrit dans le droit fil 
d'une tradition qu'elle exhibe et entend perpétuer, dans un style naturel et simple, propre à transmettre la leçon de la sagesse populaire :

Vous y verrez comment nos ayeux savoient insinuer qu'on tombe dans mille desordres quand on se plaît à ne rien faire, ou pour parler comme eux, qu'Oisiveté est mère de tous vices, \& vous aimerez, sans doute, leur manière de persuader ${ }^{7}$.

En s'effaçant ainsi, elle fait passer son discours, on le voit, pour pure transmission et démonstration, non forcément de la validité d'un contenu, mais d'un savoir ancien qui cristallise une matière (morale) et une manière (une rhétorique de l'amplification et de la variation) qu'elle aurait toutes deux assimilées.

Offrant cette fois à la duchesse d'Épernon un autre conte publié dans le même recueil, Les Enchantements de l'éloquence ou les Effets de la douceur, la conteuse le présente comme « une de ces fables gauloises qui viennent apparemment en droite ligne des conteurs ou troubadours de Provence, si célèbres autrefois». Elle affirme tenir celui-ci d'« une dame très instruite des antiquités grecques et romaines, et encore plus savante dans les antiquités gauloises » :

[elle] m'a fait ce conte quand j'étais enfant, pour m'imprimer dans l'esprit que les honnêtetés n'ont jamais fait de tort à personne, ou, pour parler comme le vieux proverbe, que beau parler n'écorche point la langue, et que souvent,

Doux et courtois langage

Vaut mieux que riche héritage ${ }^{8}$.

10 Cette première conteuse, mondaine comme elle, n'en doutons pas, aurait donc été capable d'opérer la synthèse de deux traditions, plus ou moins antiques, mais tout aussi savantes aux yeux de l'intéressée (notons que c'est la sagesse populaire française qui l'emporte quand même, pour l'auteur, sur le patrimoine des Anciens). La dame serait donc parvenue à imprimer durablement le souvenir de ce conte dans la mémoire de son élève. Ainsi, le goût des mondains pour les proverbes, rappelé dans l'autre conte, est subtilement rattaché, par sa présence dans le même recueil, à la bonne vieille tradition populaire, tandis que le rôle prépondérant des femmes dans la propagation de la culture mondaine est doublement célébré $^{9}$ : dans les deux vers cités plus haut, la conteuse fait la synthèse d'un proverbe et des moralités de deux contes de Perrault ${ }^{10}$.

11 Revenons à Finette. Mlle Lhéritier situe les événements narrés dans un passé lointain et européen (le temps des premières croisades). Le premier personnage qu'elle présente, " un roi de je ne sais quel pays d'Europe », est père de trois filles : "Ma Chronique ne m'a point appris leur veritable nom; je sais seulement que... ${ }^{11} »$. Savoir lacunaire, ce qui confirme l'ancienneté du fait rapporté. Le récit se trouve paradoxalement ancré dans une réalité et une vérité historique, en même temps que détaché d'elles. Cet effet de flou s'assortit très bien du mode de transmission du conte. Il part d'une lecture ancienne, et/ou de la trace plus ou moins fidèle laissée dans la mémoire. (On notera encore la référence, peu nette cette fois, à la double origine, orale et écrite). Quoi qu'il en soit, la lecture de la « chronique » se mue en discours oral, au moins cherche-t-on à en créer l'illusion : la narration est parsemée de nombreuses marques d'oralité. Citons pour exemple cette apostrophe à la dédicataire : «Vous, qui êtes si savante dans toutes sortes d'antiquitez, je ne doute pas, Comtesse charmante, que vous n'ayez cent fois entendu parler du merveilleux pouvoir des Fées. Le roy dont je vous parle... ${ }^{12}$ ». La conteuse, au fil du récit, adopte même une certaine naïveté de ton, comme si, retrouvant la voix maternelle de sa première éducatrice, elle s'adressait à un enfant :

N'est-il pas vray, belle Comtesse, que ce Riche-Cautele étoit un grand scelerat, \& ces

deux Princesses, de lâches \& imprudentes personnes? Je suis fort en colere contre 
tous ces gens-là, \& je ne doute pas que vous n'y soyez beaucoup aussi : mais ne vous inquietez point; il seront tous traitez comme ils meritent. Il n'y aura que la sage \& courageuse Finette qui triomphera ${ }^{13}$. fois maternant et puéril, tiennent dans un commentaire livré, cette fois, à l'issue du conte :

Voilà, Madame, la tres-merveilleuse Histoire de Finette. Je vous avouë que je l'ay brodée, et que je vous l'ay contée un peu au long [...] Vous pouvez croire, charmante Comtesse, qu'il est facile de [...] réduire [les contes] en abregé : Je vous assure que quand vous voudrez, je vous diray les avantures de Finette en fort peu de mots. Cependant ce n'est pas ainsi que l'on me les racontoit quand j'étois enfant: le recit en duroit au moins une bonne heure ${ }^{14}$.

Notons que dans Les Enchantements de l'éloquence, son récit était, à l'inverse, considérablement amenuisé :

Si je voulais, Madame, vous conter cette histoire entièrement dans les termes que les conteurs de Provence l'ont apprise à nos grands-mères, je vous dirais mille particularités étonnantes de l'adresse de Blanche, mais il est inutile; je vous dirai seulement que $[. . .]^{15}$.

Élasticité virtuelle du contage, liberté et adresse du conteur, maitrisant l'art de l'éloquence, capable d'orner le simple ou d'extraire l'essence d'un propos contourné. Dans Finette, l'auteur insiste encore sur l'ancrage dans l'enfance et, pour terminer, élargit le champ restreint de son histoire personnelle :

Je ne sçay pas si dans cet âge on vous a parlé de Finette, mais pour moy,

Cent \& cent fois ma gouvernante,

Au lieu de Fables d'animaux

M'a raconté les traits moraux

De cette Histoire surprenante [...]

Oüi, ces Contes frappent beaucoup,

Plus que ne font les faits \& du Singe \& du Loup ;

J'y prenois un plaisir extrême ;

Tous les enfants en font de même ${ }^{16}$.

Les lecteurs ne peuvent manquer d'identifier ici la double référence à La Fontaine (mort l'année où la conteuse écrit ces lignes), à ses fables et à ce désir exprimé dans l'une d'elles ${ }^{17}$ : «Si Peau d'âne m'était conté, / J'y prendrais un plaisir extrême ». À ce désir, Perrault venait de répondre l'année précédente. Il s'agit donc pour $\mathrm{M}^{\text {Ile }}$ Lhéritier, en partant de plusieurs souvenirs rassemblés et stratifiés, de mettre en évidence la richesse et les virtualités d'un héritage collectif qui surpasse celui des partisans des Anciens :

Mais ces Fables plairont jusqu'aux plus grands esprits,

Si vous voulez, belle Comtesse,

Par vos heureux talens orner de tels recits.

L'antique Gaule vous en presse :

Daignez donc mettre dans leurs jours,

Les contes ingenus, quoique remplis d'adresse

Qu'ont inventés les troubadours.

Le sens misterieux que leur tour enveloppe,

Egale bien celuy d'Esope ${ }^{18}$.

En plein cœur de la Querelle des Anciens et des Modernes, ravivée en cette dernière décennie, la conteuse se range clairement sous la même bannière que son oncle, le célèbre académicien ${ }^{19}$, et brandit les mêmes arguments. Le discours militant et 
prosélyte de $\mathrm{M}^{\mathrm{lle}}$ Lhéritier exhibe de manière obsessionnelle le patrimoine national dans plusieurs de ses Euvres meslées et invite ici à l'écriture conteuse. Cela présuppose le désir ou le fantasme selon lequel le jaillissement démonstratif d'une source enfouie propulsant la semence originelle à la surface des consciences contemporaines suffit à la propager efficacement. Plus qu'un maillon de la chaîne de transmission, l'auteur entend initier une démarche commune, elle le dit très clairement ailleurs : «je ne fais que mettre les autres en train: n'est-ce pas beaucoup faire que de marcher des premières dans des routes nouvelles $?^{20}$ ». On aura noté, dans le texte cité précédemment, l'identification totale de la voix de la conteuse à celle de l'antique Gaule, dont elle se fait, par cette audacieuse image, l'allégorie vivante, la stimulante égérie.

Perrault et sa nièce, plus que les autres, théorisent donc cette forme ancienne de récit, le conte, doté d'une force percutante, ainsi qu'en témoignerait sa survivance : «il n'y a que le merveilleux qui frappe bien vivement l'imagination ${ }^{21} »$. Intéressés par ces "récits [qui] n'avaient pour but que l'instruction des jeunes gens", ils se les approprient et, les faisant passer dans le domaine de l'écrit, les mettent au service de leur cause, en s'appuyant sur l'argument de leur valeur morale. $\mathrm{M}^{\text {lle }}$ Lhéritier explique qu'elle entend donner à voir et instruire (sans doute davantage ses compatriotes que les enfants) en recourant au conte, forme-réceptacle dotée du dynamisme de la poussée inter-séculaire dont il est le fruit. Cette instruction n'exclut en rien le plaisir, au contraire :

Quand on dit des Contes, c'est une marque que l'on n'a pas beaucoup d'affaires : on cherche à s'amuser, \& il me paroît qu'il ne coûte pas plus de les allonger, pour faire durer davantage la conversation. D'ailleurs, il me semble que les circonstances sont le plus souvent l'agrément de ces Historiettes badines ${ }^{22}$.

Pur divertissement (les auteurs aiment à le rappeler, pour se démarquer des auteurs professionnels, «écrivants » roturiers), le conte peut donc aussi convertir, et sa parole excentrique recentrer.

Mais ce retour affirmé à la tradition populaire n'est pas pure et simple régression, effet d'une nostalgie ou d'une curiosité d'anthropologue. Perrault, empruntant la voix de son fils pour offrir son recueil de contes en prose à Élizabeth Charlotte d'Orléans, justifie l'intérêt qu'ils offrent, et pas seulement pour les enfants, en cautionnant sa démarche par une référence aux grands du royaume :

À qui convient-il mieux de connaître autrement les peuples, qu'aux personnes que le Ciel destine à les conduire ? Le désir de cette connaissance a poussé des Héros, et même des Héros de votre race, jusque dans des huttes et des cabanes, pour y voir de près et par eux-mêmes ce qui se passait de plus particulier : cette connaissance leur ayant paru nécessaire pour leur parfaite instruction ${ }^{23}$.

Des arguments similaires pourront, en 1699, être également formulés par Fénelon, dans son Télémaque (l'abaissement volontaire vers les petits est nécessaire à l'éducation des princes, ainsi que savent le pratiquer les bons pères avec leurs enfants). Par ailleurs, la collecte implique aussi un élagage. On épure, on déleste les récits anciens de leur grossièreté, et on le fait savoir, pour signifier la supériorité de ces productions modernes sur les anciennes. Perrault souligne dans la préface de ses contes en vers l'immoralité de la matrone d'Éphèse et de l'histoire de Psyché, et fait état des limites qu'il s'est fixées :

J'aurais pu rendre mes contes plus agréables en y mêlant certaines choses un peu libres dont on a accoutumé de les égayer; mais le désir de plaire ne m’a jamais 
assez tenté pour violer une loi que je me suis imposée de ne rien écrire qui pût blesser ou la pudeur ou la bienséance ${ }^{24}$.

21 Action d'autant mieux fondée que le recueil est censé être produit par un enfant et entend toucher un vaste public. Quant à sa nièce, elle nuance son éloge de la tradition, de ces «fables gothiques, qui ne sont faites que pour porter aux bonnes mœurs », mais «sont souvent remplies d'histoires scandaleuses ${ }^{25}$ ». Elle précise qu'elle a réformé la conduite des sœurs de l'héroïne, dans la fable de Finette, sans suivre la tradition qui en « raconte les faiblesses odieuses avec des circonstances choquantes »:

Je croi [...] que ces contes se sont remplis d'impuretez en passant dans la bouche du petit peuple ; de même qu'une eau pure se charge toûjours d'ordures en passant par un canal sale. Si les gens du peuple sont simples, ils sont grossiers aussi : ils ne savent pas ce que c'est que la bienseance [...] Si le peuple ou les troubadours s'étaient exprimés comme nous, leurs contes n'en auraient que mieux valu ${ }^{26}$.

Grossièreté innocente, au fond, à ses yeux, liée à la naïveté populaire et rachetée par la valeur morale qui triomphe, malgré tout, dans les récits primitifs. Par ailleurs, elle fera bien la distinction, dans la même épître, entre les nobles auteurs de sa sphère d'élite et les «provinciaux vulgaires » qui n'aiment que les ouvrages remplis d'un «pompeux galimatias, qu'ils n'entendent point » :

La naïveté bien entendue n'est pas connue de tout le monde. Je ne croi pas que les nouvelles de la source des Troubadours, ni les Romances, trouvent jamais leur compte auprès de ceux qui n'ont pû sortir du caractere ordinaire que donnent certaines provinces ${ }^{27}$.

Un conteur anonyme de 1699 se moquera de cette oralité prétendue, revendiquée par ses illustres contemporains, laissant entendre avec humour que son soulignement répétitif relève d'un artifice propre au genre: "Si l'on me demande comment je sais des choses qui se sont passées dans des régions si éloignées, je répondrai qu'une fée, ou mon petit doigt me les a dites ». Ainsi s'achève le conte du Palais de la Magnificence ${ }^{28}$. Mais c'est $\mathrm{M}^{\text {me }}$ de Murat qui marquera, plus que $\mathrm{M}^{\text {lle }}$ Lhéritier, le distinguo avec la tradition gauloise et les « contes de ma Mère l'Oye » : les « fées modernes " auxquelles elle dédie ses Histoires sublimes et allégoriques (1699) sont bien différentes des anciennes, dont « les occupations étoient basses \& puériles, ne s'amusant qu'aux servantes \& aux nourrices ». Les siennes ont " pris une autre route ", n'habitent qu' "à la cour des rois ou dans des palais enchantez ", dans ces décors mondains où les contes oraux avaient depuis plusieurs décennies été placés en plus délicates bouches. Elles ne s'occupent « que de grandes choses » à côté de leurs ancêtres :

Tout leur soin consistoit à bien ballayer la maison, mettre le pot au feu, faire la lessive, remuer \& endormir les enfans, traire les vaches, battre le beurre, \& mille autres pauvretez de cette nature ; \& les effets les plus considerables de leur art se terminoient à faire pleurer des perles \& des diamans, moucher des émeraudes, \& cracher des rubis. [...] tout ce qui nous reste aujourd'huy de leurs Faits \& Gestes ne sont que des Contes de ma Mère l'Oye. Elles étaient presque toujours vieilles, laides, mal-vetuës, \& mal logées ; \& hors Mélusine \& quelques demy douzaines de ses semblables, tout le reste n'étoient que des gueuses ${ }^{29}$.

L'allusion transparente aux Fées de Perrault et aux Enchantements de l'éloquence de sa nièce n'est pas flatteuse. Voilà leur cher folklore caricaturé, renvoyé dans la crasse des chaumières, leurs nourrices et troubadours remisés au cabinet des antiquailles. $\mathrm{M}^{\mathrm{me}} \mathrm{de}$ Murat tient à révéler ses sources écrites (Les Facétieuses nuits de Straparole, de nombreuses fois réimprimées), précisant que ses contemporaines s'y sont copieusement abreuvées : «Les Dames qui ont écrit jusques icy en ce genre, ont puisé 
dans la même source au moins pour la plus grande partie ${ }^{30}$ ». Il s'agit non seulement pour elle de se soustraire à une éventuelle accusation de plagiat (elle vise assez explicitement $\mathrm{M}^{\mathrm{me}} \mathrm{d}$ 'Aulnoy), mais peut-être, plus insidieusement, de railler la

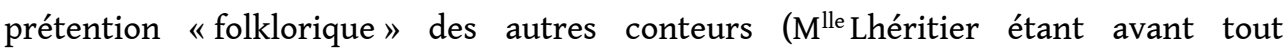
concernée), qui n'avouaient pas de telles sources littéraires. (Notons que Perrault, curieusement, est mis hors de portée de ses réflexions, alors qu'il s'est lui aussi inspiré de Straparole). L'aveu que $\mathrm{M}^{\mathrm{me}}$ de Murat fait de ses modèles ne lui retire rien de sa création: «Quelque mediocre [sic] que soient les ouvrages que l'on donne au public, l'on sent toûjours pour eux un amour de pere qui oblige d'en justifier la naissance ». (On peut se demander si cette phrase, où elle gomme bizarrement sa féminité, ne viserait pas cette fois malicieusement l'académicien, qui savait utiliser sa paternité non pour « justifier la naissance » de ses contes en prose, mais pour la déplacer sur son fils). Un autre conteur, Nodot, dans l'épître dédicatoire de son Histoire de Mélusine ${ }^{31}$, explique en 1698 que « cette héroïne [lui] a inspiré de composer un corps d'histoire des merveilles qu'elle a faites à la veuë de toute l'Europe. » Il a donc "recüeilli tous ces événemens fameux », croyant « faire une chose agréable au public de ramasser ce que l'histoire et la tradition ont conservé de Mélusine ». De cette cueillette peut naître ensuite le conte, moyennant un travail d'érudition, parfois souligné, ou tout simplement d'une réécriture, qui lui confère une valeur ajoutée : Nodot précise qu'il n'a pas laissé ces événements "aussi nuds qu'ils le sont dans les chroniques", mais qu'il leur a donné «les ornemens qui peuvent leur convenir ». Ornements qui esthétisent l'œuvre : c'est tout un parcours de l'originel à l'original qui est ici tracé.

Bien davantage que les conteurs qui leur emboîteront le pas, les pionniers prennent donc la peine d'exposer leur démarche, dans les préfaces ou les moralités, ou encore au détour d'une intervention de narrateur. Si leurs contes sont souvent, à leurs yeux, les recueils d'un patrimoine national ou européen, folklorique ou littéraire revivifié chansons des troubadours, épopée médiévale, récits courtois auxquels le conte de fées avait emprunté son personnage emblématique, les premiers contes sont bien ancrés dans leur époque, même ceux qui se veulent issus d'une tradition populaire, et plusieurs attestent d'une esthétique néo-précieuse. Ils font grand usage de maximes, proverbes, qui étaient également prisés dans les salons: proverbes dont ils se prétendent parfois une sorte d'expansion $\left(\mathrm{M}^{\text {me }}\right.$ Lhéritier présente les siens comme des " historiettes au sujet des proverbes ${ }^{32}$ » à sa dédicataire qui les aime), maximes dont ils proposent l'illustration. (Le goût pour les proverbes passera certes rapidement, mais on pourra moins vite renoncer à souligner la valeur morale des contes). À ces influences perceptibles ou revendiquées, il faut bien sûr ajouter les interférences nombreuses avec d'autres genres, littéraires, cette fois (d'abord celle, prioritaire, de la nouvelle galante, à la mode pendant le dernier demi-siècle) et avec d'autres formes (opéra, théâtre, poésie pastorale, lettres...). À géométrie variable, comme le roman, les contes de fées se prêtent par ailleurs très tôt à l'encastrement de ces petites formes et de ces histoires secondaires qui les complexifient. À plus d'un titre (origine, structure, thèmes, motifs...), les contes de fées sont donc bel et bien des recueils, ils sont travaillés par ce que l'on pourrait appeler une « esthétique du recueil».

Contes encastrés, contes regroupés

26 La pratique ludique du contage mondain, en vigueur dans les salons et à la cour depuis plusieurs décennies, n'est pas seulement commentée par les textes préfaciels des premiers auteurs, à des fins idéologiques et militantes. Elle se trouve reproduite et mise 
en scène dans les romans de l'époque, et d'autant plus que plusieurs conteurs étaient aussi et avant tout des romanciers. Il s'agit de mimer, de près ou de loin, un usage social : le contage, qui relève d'une pratique communautaire et d'une mode: on veut être diverti, on aime les contes de fées, on en réclame à quelqu'un qui en connaît, ou on en propose soi-même.

Le premier conte de fées littéraire fait timidement irruption en 1690 dans un roman de $\mathrm{M}^{\mathrm{me}} \mathrm{d}$ 'Aulnoy, l'Histoire d'Hypolite comte de Duglas. Elle réutilisera cette technique de l'insertion à plusieurs reprises, mais auparavant, Perrault aura publié ses premiers

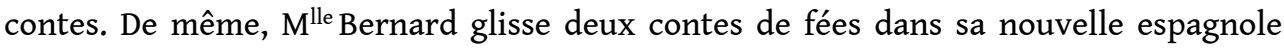
Inès de Cordoue (1696). Ils apparaissent dès l'ouverture. L'insertion du Prince Rosier et de Riquet à la houppe produit plusieurs effets. Le plus évident est un effet de réel instantané. L'action, qui se situe en Espagne, commence peu de temps après le mariage de Philippe II et d'Élizabeth de France (1560). La distance historique et spatiale est soudain abolie par l'insertion du conte, qui a été préparée dès les premières lignes: "[la] cour était devenue galante et les divertissements n'en étaient pas bannis ${ }^{33}$ ". L'auteur note en effet l'influence d'un esprit français déterminant les goûts de la reine pour « la conversation, [...] les vers, la musique et tout ce qui avait du rapport à la galanterie. » Chaque après-midi, la reine tient une sorte de salon avec quelques dames, où elle propose, "pour se faire un amusement nouveau, d'imaginer des contes galants ». Proposition qui ravit tout le groupe :

On convint de faire des règles pour ces sortes d'histoires dont voici les deux principales: que les aventures fussent toujours contre la vraisemblance, et les sentiments toujours naturels. On jugea que l'agrément de ces contes ne consistait qu'à faire voir tout ce qui se passe dans le cœur et que, du reste, il y avait une sorte de mérite dans le merveilleux des imaginations qui n'étaient point retenues par les apparences de la vérité ${ }^{34}$.

S'élabore ici une esthétique du conte mondain. Les règles à suivre sont moins des règles de jeu que des canons littéraires. Elles ont un point commun avec celles de la nouvelle historique, à cette différence près du merveilleux : le naturel des sentiments est une donnée partagée par les deux genres, qui font en outre circuler - les critiques l'ont noté - les mêmes valeurs et expriment les mêmes désirs, les mêmes hantises. L'intrigue du conte, plus ramassée, opère comme un effet de loupe par rapport à l'ensemble du corpus et dénote une vision de l'amour totalement désabusée: à trouver leur aboutissement dans le mariage, les rêves amoureux des personnages s'y brisent. Une fois que sont levés les obstacles externes à la réalisation de leurs vœux, les individus se heurtent à d'autres, plus puissants : les tréfonds de leur propre cœur, secoué par des pulsions confuses et inavouables ${ }^{35}$. Aucune autre issue possible ne se laisse alors entrevoir que la destruction et le silence : repli dans la retraite pour les personnages de la nouvelle ou, pour le prince Rosier, retraite symbolique et régression végétative ${ }^{36}$. La pulsion de mort œuvre souterrainement, dans ces deux contes comme dans la nouvelle, et plus encore dans Riquet à la houppe où c'est, cette fois, au propre que les amants enterrent leurs illusions ${ }^{37}$. Le merveilleux ne serait ici qu'une fenêtre ouverte un bref moment sur un idéal amoureux et le rêve de désirs comblés, fenêtre bien vite refermée sur l'échec. L'insertion de contes donnant une image aussi négative de la passion amoureuse, contes qui se finissent mal tous deux, sonne comme un avertissement sinistre et fait figure de tragique prémonition. Les contes seraient porteurs d'un savoir inconscient sur le destin de leurs protagonistes censés les avoir inventés. Loin d'être le substrat de la sagesse populaire, le conte est ici, comme le rêve, une plongée dans 
l'univers intime, un révélateur potentiel, même si cette potentialité n'est pas confirmée par leurs auteurs fictifs, encore innocents ou aveugles. L'échec de l'amour, dont les précédents romans de Catherine Bernard sont autant d'illustrations, semblent signer la défaite du dessein que leur auteur a formulé dans l'avertissement précédant Éléonor d'Yvrée (1687) et rappelé dans Le Comte d'Amboise (1699) : « ne faire voir que des amants malheureux pour combattre, autant [que] possible, le penchant qu'on a pour l'amour ${ }^{38}$ ». La vertu pédagogique des contes, souvent alléguée par les autres auteurs, ne pourrait donc pas opérer sur les principaux intéressés. Après lecture de la nouvelle qui cadre le conte, celui-ci se recharge alors d'une vérité universelle, le principe posé par Catherine Bernard prend valeur mythique, dans un effet-retour. La publication des contes hors de leur encadrement, parti pris éditorial qui fut très longtemps en vigueur, dépouille les uns et les autres d'une part de leur sens.

29 L'insertion des contes dans la nouvelle nécessite une motivation interne à l'histoire, le conte ainsi fictionnalisé se dote d'un enjeu : le conte, objet de divertissement, devient objet de séduction et prend place dans une stratégie alimentée par la rivalité amoureuse. Inès est désignée par tirage au sort, elle conte Le Prince Rosier, et le lendemain, Léonor «se prépara à conter une fable et n'oublia rien pour l'emporter, s'il se pouvait, sur Inès ${ }^{39}$ \%. Rien ne permet de savoir si des motivations comparables pouvaient toujours diriger les conteuses dans les salons et les cercles mondains de époque, quoiqu'une très probable concurrence soit perceptible dans les déclarations de $\mathrm{M}^{\text {me }}$ de Murat rapportées précédemment. Toujours est-il que l'insertion du conte de fées dans la fiction, établissant une passerelle réaliste entre l'univers fictif et l'univers réel, a pour effet de surfictionnaliser le conte et, à l'inverse, de défictionnaliser quelque peu la nouvelle, par un système de vases communicants.

30 Entre temps le genre féerique a acquis son autonomie, puisque le premier tome des Contes des fées (1697) de $\mathrm{M}^{\text {me }} \mathrm{d}$ 'Aulnoy et le recueil de Perrault, publié la même année, sont composés de contes autonomes et simplement juxtaposés, sans lien narratif entre eux. Plusieurs de leurs proches contemporains exploiteront cette formule simple et sa structure en éventail : en 1698, Mailly pour ses Illustres fées, contes galans, $\mathrm{M}^{\text {me }}$ de Murat pour ses Contes de fées et Nouveaux contes des fées ou encore, l'année suivante, ses Histoires sublimes et allégoriques. Dès le troisième tome du recueil de $\mathrm{M}^{\mathrm{me}} \mathrm{d}$ 'Aulnoy (1697), les contes sont insérés dans des nouvelles, et l'ensemble est présenté par un récit liminaire dans lequel la conteuse se met en scène elle-même, en compagnie d'aristocrates qui connaissent ses contes et lui envient son talent. L'encastrement de contes de fées dans des cadres plus ou moins fictionnels, dès lors, fait sentir que les contes ont acquis, de toute évidence, une fonction sociale. Ils jouent un rôle dans la vie des personnages de la fiction qui déborde largement le cadre du simple passe-temps : dans les nouvelles, le savoir-conter est souvent un formidable outil de diversion pour celui qui le possède et pas seulement un moyen de divertissement pour les auditeurs. Il lui confère le pouvoir de parvenir à ses fins, qui peuvent être tout autres que celles qu'il revendique. Les personnages conteurs, toujours publiquement asservis à un auditoire auquel il faut plaire, ont sur lui, à son insu, une emprise considérable ${ }^{40}$.

De l'homogène à l'hétéroclite, ou le virage du siècle

31 Le recueil, considéré par les libraires comme un emballage de prédilection pour les petites formes, offre aux premiers conteurs le moyen de donner une cohérence à une multiplicité. Il devient dès le troisième volume des Contes des fées de $\mathrm{M}^{\mathrm{me}} \mathrm{d}$ 'Aulnoy ce meuble à tiroirs bien ordonné, soumis aux impératifs pseudo-réalistes d'un découpage 
de lecture : les contes y sont lus, le plus souvent, par des protagonistes qui scindent les plus longs contes en épisodes conçus à la dimension crédible d'une lecture orale et collective. Ces recueils sont soumis à une esthétique de l'alternance rythmique (nouvelle-cadre/conte de fées). En 1699, le recueil de contes de fées s'ouvre à une diversité générique autre que celle des CEuvres meslées de $\mathrm{M}^{\text {lle }}$ Lhéritier (où prose et poésie étaient associées) : est publié anonymement le Recueil de contes galans, déjà cité, qui contient trois contes de fées, placés après une nouvelle qui n'a aucun lien avec eux et reprend l'argument d'un conte de $\mathrm{M}^{\mathrm{me}} \mathrm{d}$ 'Aulnoy. La même année, $\mathrm{M}^{\mathrm{me}}$ de Murat, dans son Voyage de campagne, n'encastre qu'un seul conte merveilleux, Le Père et ses quatre fils, parmi d'autres pièces de genres variés. Présentant ce conte, elle en souligne la différence :

Il n'y a point de ce merveilleux qu'on voit dans tous les autres contes de cette espèce [...] J'ai voulu en retrancher les Fées, pour voir si je pourrois rendre mes amans heureux, sans le secours de ces bonnes dames, qui sont justement les dieux de la machine que les Anciens condamnent ${ }^{41}$.

$\mathrm{M}^{\mathrm{me}}$ Durand, en 1702, suit ce mouvement de diversification avec Les Petits Soupers de l'été de l'année 1699, de même que $\mathrm{M}^{\mathrm{me}} \mathrm{d}$ 'Auneuil, qui publie, toujours en 1702, La Tyrannie des fées détruite et ses Nouvelles diverses du temps. Le premier ouvrage est un recueil de contes juxtaposés, le second exploite d'une manière assez originale la technique de l'encastrement. Il s'agit de petites brochures, bien éloignées, par leur taille, des recueils de plusieurs centaines de pages parus jusqu'alors. Elle en donnera quatre livraisons successives entre 1702 et 1703.

Arrêtons-nous sur ces «nouvelles ». Le choix d'une telle désignation est révélateur. Les contes publiées par les prédécesseurs se piquaient tous de nouveauté et parfois de manière saugrenue (Préchac se distançant du troupeau en prétendant offrir au public de 1698 des Contes moins contes que les autres). En optant pour un tel substantif, $\mathrm{M}^{\text {me }}$ d'Auneuil fraye avec deux autres types de production. D'abord celui qui est concomitant dans la fiction narrative. Ensuite, donner des nouvelles « du temps », c'est en général l'affaire de la presse, des périodiques et des gazettes. Plus simplement enfin, au quotidien, le terme évoque la banale conversation, parfois avide de bons mots, de potins plus ou moins libres. Le titre de $\mathrm{M}^{\text {me }} \mathrm{d}$ 'Auneuil annonce donc implicitement une nouvelle manière de faire converger l'oral et l'écrit (celle des premiers conteurs avait été d'exhumer les contes du passé, de la mémoire des nourrices ou de la tradition, tout au moins de prétendre le faire). Il faut donc à présent que ces «nouvelles" soient contemporaines, localisées dans la sphère géographique des lecteurs. La détermination «nouvelles du temps » appose sur elles une estampille de vérité supérieure à celle des rares premiers contes appelés aussi «nouvelles ${ }^{42}$ ». Les siennes contiennent un ensemble de lettres qu'un homme envoie à une dame. Il s'en explique ainsi :

Ne croyez pas, Madame, que les plaisirs de Paris me fassent oublier que vous m'avez ordonné de vous envoyer tout ce qui se fera de nouveau dans cette grande ville. Je laisse au Mercure galant le soin de vous instruire des conquêtes de notre grand Monarque, et des activités éclatantes de ce digne rejeton de sa gloire comme de son rang. Pour moi je me charge de vous apprendre ce qui se passe dans les ruelles des dames, et dans le cabinet des muses ${ }^{43}$.

La grande histoire est reléguée dans les périodiques; au rédacteur de la lettre, il reste la petite. Secrets d'alcôves et pièces galantes seront donc au menu d'une collecte de nouveautés, du moins tel est ce qui s'annonce, avec ce que cela implique de décousu, de disparate, ceux-là mêmes de la vie. 
rons donc ces prétendues « nouvelles ». La première, La Princesse des pretintailles, est un conte de fées. Ce sera le seul de toutes les brochures. On y évoque la mode parisienne contemporaine, en attribuant l'origine d'un détail vestimentaire à une féerie de pacotille. La pièce suivante est un conte allégorique, intitulé L'Origine de l'occasion. On y développe celle d'une expression, « saisir l'occasion par les cheveux " (l'allégorie de l'Occasion coiffée d'une mèche unique est loin d'être nouvelle) et on en profite pour illustrer un proverbe: "une occasion perdue ne se rattrape plus». Vient alors une anecdote narrant l'aventure très contemporaine survenue à la marquise d'Aubignac, qui faillit se noyer dans la Seine. Suit une pièce de vers, produite, dit-on, par l'homme qui la sauva de l'eau. Pour justifier sa présence ici, le rédacteur explique qu'il a été chargé par son auteur de la transmettre à la belle rescapée. Une fois ce secret divulgué, la lettre s'achève sur un Essai de l'histoire de Perse, tiré de Josèphe et Philon! Ces différents morceaux, fort hétérogènes, sont reliés par de rapides transitions, de plus en plus lâches, et le seul rapport qu'ils entretiennent avec l'actualité est leur croisement prétendu avec le vécu du rédacteur qui semble avoir oublié son projet de départ, tant la temporalité est audacieusement distendue, et de manière de plus en plus visible (les événements rapportés dans la dernière histoire se passent en l'an 243). Le protagoniste s'en explique ainsi : il vient de recevoir cette histoire d'un ami. Voilà à quoi est réduite l'actualisation. Dès lors, on le voit, tout est possible. N'importe quel genre peut trouver ici sa justification, à condition que ce «n'importe quoi » soit bref. Dans la brochure suivante, de novembre, le rédacteur présente à sa destinataire un portrait composé par son père :

Souffrez que je vous envoie le portrait que vous m'avez demandé fait par feu mon père. J'ai été contraint d'ajuster sa prose à la moderne, quoi que pour ce tems-là elle fut assez estimée. Je connais votre vivacité, la longueur de ces phrases et ces conséquences tirées du sujet, vous auroient furieusement dérangées ${ }^{44}$.

Le père est mort, mais la lettre est nouvelle, même si cette nouveauté était ici moins que partout ailleurs nécessaire; elle constitue une trahison, une transgression de la demande émanant de la destinataire. Cette incohérence ramène cette dernière à sa vraie place, celle d'alibi pour la publication, et la nouveauté, affichée en titre, à celle de publicité éditoriale. Moyennant un fil ténu de narration, qui se colore de galanterie (car le destinateur de la lettre en profite ici ou là pour courtiser sa destinataire, l'invitant à méditer sur ces histoires et à imiter, par exemple, les héros qui savent si bien tirer profit de l'occasion), le recueil est devenu fourre-tout, bric-à-brac hétéroclite. Anecdotes, essais, fausses traductions, contes de genres divers, lettres, prose et vers s'y succèdent. La formule épistolaire, jamais encore utilisée en récit-cadre d'un conte de fées, s'avère une recette bien commode pour assurer un lien minimal.

D'autres recueils non féeriques publiés à l'époque recouraient déjà à un semblable encadrement épistolaire. En 1699 était parue la seconde édition, augmentée, des Lettres nouvelles de M. Boursault ${ }^{45}$. L'ajout, mentionné explicitement dans le titre, concernait entre autres des contes et des épigrammes. Trois contes très brefs, d'inspiration gauloise, y étaient introduits par une lettre anonyme spécifiant que l'on cédait à la pression du destinataire. Le Journal des Sçavants salue la parution de cette réédition par un jugement qui occulte les contes, pour saluer uniquement le mélange de poésies aux lettres. "Leur diversité fait qu'on les lit dans qu'on se puisse jamais ennuyer ${ }^{46}$ ». Cette qualité, célébrée en son temps par La Fontaine est, on le voit, une valeur toujours actuelle et un critère toujours porteur. 
Si un titre comme La Princesse des prétintailles peut encore s'inscrire dans la littérature féerique des dernières années, L'Origine des cornes ou l'Inconstance punie, autre conte des Nouvelles diverses $d u$ temps, relève davantage d'une veine populaire légère qui est celle des contes en vers de La Fontaine, veine toujours vivace à l'époque, et notamment dans les recueils de bons mots et de chansons ${ }^{47}$.

9 Le recueil, en 1702, prend donc de plus en plus la forme du pot-pourri. Si l'on manifeste encore, cette année-là, le souci d'une apparente cohérence, elle est de pure forme chez $\mathrm{M}^{\mathrm{me}} \mathrm{d}$ 'Auneuil. Que le texte d'encadrement existe ou non, les mixtures des petites formes sont, au tournant du siècle, de moins en moins homogènes. De ce creuset, le conte de fées ne sort pas indemne. Il perd tout d'abord l'exclusive; ensuite la promiscuité des textes de types différents semble le contaminer. Le genre féerique s'y métamorphose au contact d'autres formes littéraires. Ses modes d'écriture se dévoyant, le recours aux stéréotypes féeriques tend de plus en plus vers la caricature. Le genre est mûr, dès lors, pour la parodie voyante, d'où il tirera une énergie vitale qui lui permettra de réaffirmer son identité. Cette force, déjà en germe chez les premiers conteurs, a vocation à s'affirmer davantage au fur et à mesure que les recettes sont réutilisées. Les contours du conte, à l'origine plutôt imperméables à leurs récits d'encadrement - au moins en apparence, hormis quelques jeux spéculaires appuyés çà et là - deviennent ostensiblement poreux, en ces années de transition, et leurs univers de référence se recouvrent de plus en plus ${ }^{48}$.

La dialectique éditoriale du conte et du recueil

Reste à se pencher, pour terminer, sur les méandres de la diffusion des contes écrits. Le choix de l'œuvre conteuse de Perrault permet de mettre en évidence la dialectique éditoriale du conte et du recueil. En effet, cette œuvre inaugure trois des types possibles de publication qu'ont connu les contes (recueil collectif de genres divers, conte seul, recueil personnel de contes juxtaposés). Cet exemple est à la fois significatif et très particulier, car ces textes auront un destin qui sera, en partie au moins, celui de plusieurs contes de fées de la génération, mais opérant sur un parcours beaucoup plus rapide que les autres (en majorité célèbres eux aussi pourtant à leur époque, mais relégués ensuite - et pour longtemps - dans l'oubli).

La Marquise de Salusses ou la Patience de Griselidis, nouvelle, lue à l'Académie française le 25 août 1691 après une distribution de prix d'éloquence et de poésie qui récompensent entre autres sa nièce, bénéficie des hommages du public. La nouvelle est aussitôt publiée par J.-B. Coignard, libraire du roi et de l'Académie, au sein d'un recueil des pièces présentées en la circonstance. La même année, le même libraire reçoit un privilège pour une édition séparée de la nouvelle. En novembre 1693, le Mercure galant publie Les Souhaits ridicules. L'année suivante, ce conte est repris chez la Veuve Coignard, avec Griselidis, tous deux légèrement remaniés. S'y trouve ajouté le conte de Peau d'âne, qui a peut-être paru séparément dans les derniers mois de 1693, ainsi que le suppose Gilbert Rouger, se fondant sur le fait que l'éditeur précise qu'il s'agit d'une seconde édition ${ }^{49}$. Toujours en 1694, le premier tome du Recueil de pièces curieuses et nouvelles tant en prose qu'en vers, célèbre contrefaçon publiée à La Haye chez Moetjens, fait figurer ces trois textes parmi les 120 pièces dont le volume est composé. Coignard, en 1695 , lance sur le marché une "quatrième édition", à laquelle une préface est ajoutée (ce qui pourrait encore une fois laisser supposer une édition en livret autonome entre la "seconde » et celle-ci). Le Mercure galant de février 1696 donne une première version de La Belle au bois dormant, que s'empresse de reproduire le recueil Mœtjens 
(tome $\mathrm{V}$, première partie). Le 28 octobre $1696 \mathrm{P}$. Darmancour reçoit un privilège pour ses Histoires ou contes du temps passé, avec des moralités, chez Claude Barbin, futur éditeur de plusieurs des conteurs de cette génération. Le succès de l'ouvrage, paru en 1697, est tel qu'il le réimprime la même année, pendant qu'une contrefaçon circule, et le recueil Mœtjens ne tarde pas, une fois de plus, à imprimer ces nouveaux contes (tome V quatrième partie). Le ballet des rééditions des contes en prose, chez Barbin, se poursuivra jusqu'en 1707, date où le nom de Perrault figure enfin en couverture, et d'autres éditeurs se relaieront pendant toute la première moitié du XVIII ${ }^{\mathrm{e}}$ siècle, tandis qu'on délaissera les contes en vers. Il faudra attendre 1747 pour que Les Amusemens de la campagne et de la ville tirent de l'oubli Les Souhaits ridicules, et la lointaine date de 1881 pour que l'ensemble des contes de Perrault fasse l'objet d'un regroupement.

Perrault est le seul auteur de contes qui bénéficie dans un laps de temps aussi rapproché de ces diverses formes de publication. Cela s'explique bien sûr par le succès immédiat de ses contes, favorisé par la réputation que lui avaient valu sa carrière et son statut d'académicien. La deuxième particularité de sa production est que ses recueils de contes s'épaississent au fur et à mesure de leur publication, phénomène explicable par les mêmes raisons : on peut se permettre de ne pas attendre qu'un corpus définitif soit constitué si l'auteur est suffisamment connu pour que l'on utilise le nouveau en vendant l'ancien.

43 À plusieurs reprises, la première publication de quelques-uns des premiers contes de Perrault est réalisée au sein d'un recueil hétérogène, qui leur confère à la fois une existence littéraire, une originalité et une reconnaissance, et constitue une piste de lancement pour leur diffusion, celle-ci étant en outre encouragée par les éloges fournis en accompagnement: le recueil de pièces variées de l'Académie émane d'une autorité suprême en matière de littérature (tous les auteurs ne pourront certes pas disposer d'un tel support), mais le Mercure galant est un appréciable outil de propagande en direction des publics mondains et de la Cour. Favorable aux Modernes, il saluera avec enthousiasme la publication des contes de la première génération, à défaut de les éditer tous. Une fois le lectorat créé et testé, un corpus d'au minimum deux petites pièces suffit à constituer une somme. C'est alors qu'un recueil d'auteur - recueil homogène peut exister, sa viabilité étant confirmée et assurée. Les conteurs qui succéderont à Perrault n'auront plus à suivre toutes les étapes d'un tel parcours, le succès des premiers contes leur en fera économiser quelques-unes, en même temps que le prestige qu'ils n'ont pas leur interdira l'accès à d'autres. Les auteurs devront réunir davantage de textes (au moins trois pour chacun des recueils de contes juxtaposés de $\mathrm{M}^{\mathrm{me}} \mathrm{de}$ Murat ${ }^{50}$ ) et il faudra en outre que ces derniers aient une dimension plus considérable, condition impérative pour espérer publier un conte unique (ainsi en est-il des deux romans merveilleux de Nodot, qui représentent une exception, et pas seulement à cet égard). De même, il ne sera plus besoin de préfaces ou d'avertissements aux lecteurs, la dédicace à une personne de qualité, proche du pouvoir, voire la simple référence au goût du temps apporteront une caution suffisante aux contes. Jamais, dans les premiers temps, ne paraissent de recueils de contes collectifs en première édition, contrairement au désir exprimé par $\mathrm{M}^{\mathrm{ll}}$ Lhéritier dans son introduction à Marmoisan ou l'innocente tromperie, nouvelle héroïque et satirique, dédiée à $\mathrm{M}^{\text {lle }}$ Perrault. L'auteur, complice de la mystification entretenue par Perrault sur la paternité de ses contes, prétendus composés par " un enfant ", son fils (qui a 19 ans à la date de publication des contes en prose), suggérait à sa dédicataire de transmettre Marmoisan à son frère : « vous jugerez ensemble si cette fable est digne d'être placée dans son agréable recueil de contes ${ }^{51}$ ". 
Par ailleurs, « il n’y a point de merveilleux par les enchantements dans Marmoisan [...], tout s'y passe dans l'ordre naturel ${ }^{52}$ ", ainsi que le souligne $\mathrm{M}^{\text {lle }}$ Lhéritier. Une telle diversité générique associée à un regroupement d'auteurs n'était peut-être pas une formule bien désirable, dans les tout débuts de la production féerique; on ne la trouverait assumée que bien plus tard, en 1745, avec les productions de Caylus et les recueils du Bout-du-Banc ${ }^{53}$.

Les premiers contes de fées connaitront des vies nouvelles dans des florilèges de pièces de genres extrêmement variés (tels que les diverses Bibliothèques de campagne). La pratique du pêle-mêle générique propre aux petits genres (autres que les contes de fées, mais dont leurs recueils avaient eux-mêmes hérité au tournant du siècle) ne cesse pas au cours du XVIII ${ }^{e}$, bien au contraire, elle se développe encore avec l'essor des périodiques. La consommation rapide est toujours encouragée par les éditeurs: si les lecteurs de 1700 ont à leur disposition Le Porte-feuille galant, contenant les entretiens, des caffez et autres ouvrages mêlez de prose et de vers (1700), ceux de 1805 peuvent se fournir au Porte-feuille galant, recueil amusant et varié, dédié aux dames, qui contient des chansons, bons mots, fables, charades, bouts-rimés, madrigaux. Le plaisir est une divinité immortelle et toujours célébrée. Auteurs et éditeurs continuent de miser sur les attentes d'un public avide de divertissement, et ils le signifient clairement dans les titres qu'ils arborent: il n'est qu'à constater le nombre de ces Amusements de la campagne et autres déclinaisons, tout au long du XVIII ${ }^{\mathrm{e}}$ siècle ${ }^{54}$.

Si les premiers éditeurs de contes de fées étaient soucieux de profiter d'une mode, leurs successeurs auront parfois à cœur de transmettre, une fois celle-ci évaporée, un patrimoine menacé de disparition. L'année 1717 voit la publication d'un premier Cabinet des fées ${ }^{55}$. Le titre est repris, avec modification, en $1731^{56}$ et en $1754^{57}$, mais par des éditeurs distincts et à des adresses différentes. Le Chevalier de Mayer, de 1785 à 1786, se lance dans une vaste entreprise de récolement, en opérant toutefois une sélection dans la production énorme des contes écrits depuis la fin du XVII ${ }^{\mathrm{e}}$ siècle. Il en ressortira 41 volumes qui constitueront la plus vaste anthologie de contes jamais publiée, et qui formeront son Cabinet des fées ou collection choisie des contes des fées et autres contes merveilleux. Ce dernier avatar de la publication en recueil au xvIII siècle, sous ses diverses manifestations, va donc dans le sens apparent d'un resserrement générique plus ou moins élastique, et rend hommage à un genre dont il explore une partie des richesses, montrant sa diversité, ses prolongements variés ou mettant à l'honneur certains de ses antécédents supposés (Les Mille et Une Nuits, quelques-unes des imitations du recueil de Galland, ainsi que les Fables indiennes de Bidpaï et de Lokman figurent dans le Cabinet des fées de Mayer à coté des féeries, les contes licencieux ou sentis comme trop satiriques étant exclus.) En fonction du succès que le genre aura rencontré au fil du temps, les rééditions successives de recueils d'auteurs s'ajouteront aux reprises de contes (à l'identique ou non) dans les recueils de pièces plus ou moins hétérogènes et d'auteurs différents.

D'autres éditeurs, loin de manifester semblable ambition encyclopédique, infligeront aux contes un tout autre destin. Les plus célèbres d'entre eux, réécrits, raccourcis, mutilés, se verront dépouillés de leurs moralités, de leurs encadrements narratifs et de leurs péritextes. Détournés au profit d'un autre lectorat, enfantin celui-là, ou rendus aux livrets de la Bibliothèque bleue d'où certains pionniers les avaient tirés, ils retourneront à l'anonymat pour de longs siècles, constituant ici ou là d'autres recueils, au bon vouloir des éditeurs. 


\section{NOTES}

1. Donneau de Visé s'en occupe jusqu'en 1710, puis Charles Dufresny de la Rivière prend le relais jusqu'en 1713. Le Mercure galant paraît d'abord trimestriellement, puis mensuellement, et chaque volume compte entre 200 et 400 pages. La collection totalise 571 volumes.

2. «Cet ouvrage est un recueil fidelle de tous les termes et de toutes les phrases dont l'Éloquence et la Poésie peuvent former des éloges » (Epistre au Roy, tome 1, première édition, 1694).

3. Villiers Pierre (abbé de), Entretiens sur les contes de fées, et sur quelques autres ouvrages du tems. Pour servir de préservatif contre le mauvais goût, Paris, J. Colombat, 1699, 2e entretien, p. 69.

4. Le verbe « récolter » n'apparaît qu'au milieu du XVIII ${ }^{\mathrm{e}}$ siècle.

5. Ses mémoires manuscrits le révèlent.

6. CEuvres meslées de Mademoiselle L'H***, Paris, Guignard, 1695, p. 229-230.

7. Ibid., p. 230-231.

8. Ce texte est publié dans l'édition des Contes de Perrault par G. Rouger, Classiques Garnier, 1967, p. 239-240.

9. Voir l'article de Marc Fumaroli : «Les Fées de Charles Perrault ou De la littérature » (Le Statut de la littérature, Mélanges offerts à Paul Bénichou, Droz, Genève, 1982), p. 171. Les Enchantements de l'éloquence y sont étudiés conjointement, et leur signification allégorique mise en évidence.

10. "Quelque grand que soit l'avantage / De jouir d'un riche héritage / [...] L'industrie et le savoir faire / Valent mieux que des biens acquis. " (Le Maître chat ou le Chat botté). «Les Diamants et les Pistoles / Peuvent beaucoup sur les Esprits ; / Cependant les douces paroles / Ont encor plus de force, et sont d'un plus grand prix. » (Les Fées).

11. Ouvr. cité, p. 233-234.

12. Ibid., p. 240.

13. Ibid., p. 260.

14. Ibid., p. 294.

15. Ouvr. cité, p. 245.

16. Ouvr. cité, p. 296-297.

17. Le Pouvoir des Fables, VIII, 4.

18. Ibid., p. 297-298.

19. Voir la préface des trois contes en vers de Perrault, publiée pour la première fois dans l'édition de 1695 . L'auteur y cite, par ailleurs, quelques vers de sa nièce faisant l'éloge de ses contes : échange de bons procédés.

20. Lettre à Madame D. G**, Euvres meslées, Guignard, Paris, 1695, p. 318.

21. Ibid., p. 300.

22. Ibid., p. 294-295.

23. Dédicace « À Mademoiselle » (Contes, éd. citée, p. 89-90).

24. Ibid., p. 6-7.

25. Lettre à Mme D. G**, Cuvres meslées, p. 312.

26. Ibid., p. 312-314.

27. Ibid., p. 317. 
28. Recueil de contes galans, à Paris, au Palais chez Médard, Michel Brunet, 1699.

Privilège du 12 janvier 1698, registré le 26 février 1698, achevé d'imprimer le 10 février 1699. Il existe un exemplaire de ce recueil peu connu à la bibliothèque de l'Arsenal $\left(8^{\circ}\right.$ BL 18838). M.E. Storer émet l'hypothèse, à mon sens contestable, que son auteur soit Mailly.

29. «Épître aux fées modernes », Histoires sublimes et allégoriques, 1699, p. II-III.

30. « Avertissement ", ibid.

31. Nodot François, Histoire de Mélusine, tirée des chroniques de Poitou, et qui sert d'origine à l'ancienne maison de Lusignan, Paris, Barbin et Mœtte, 1698.

32. L'Adroite Princesse, p. 230.

33. Bernard Catherine, Euvres, tome 1, Romans et Nouvelles, par Franco Piva, SchenaNizet, Fasano-Paris, 1993, p. 344.

34. Ibid., p. 347-348.

35. Voir la présentation du roman par Franco Piva, p. 325-339.

36. Après avoir échappé au maléfice qui l'a réduit à l'immobilité, état dans lequel il goûtait cependant les douceurs de l'amour naissant, il finit par réclamer aux fées de redevenir rosier, pour se soustraire à la persécution d'une épouse naguère tant désirée. 37. Le difforme Riquet, trompé par une femme qu'il a contrainte au mariage sans en être aimé, la condamne, dans le palais souterrain qu'ils habitent, à confondre à jamais époux haï et amant, en donnant à ce dernier sa propre apparence.

38. Ibid., p. 239.

39. Ibid., p. 357.

40. Je renvoie à mon ouvrage, Les Contes et les nouvelles de Madame d'Aulnoy - L'imaginaire féminin à rebours de la tradition, Droz, Genève, 1998. La première partie, consacrée aux récits-cadres (p. 31-91), étudie les divers types d'encastrement, leurs effets et implications idéologiques. Voir également Mainil Jean : Madame d'Aulnoy et le rire des fées - Essai sur la subversion féerique et le merveilleux comique sous l'Ancien Régime, Paris, Kimé, 2001.

41. Voyages extraordinaires, songes, visions et romans cabalistiques, tome 29, Amsterdam, Garnier, 1788, p. 68.

42. Perrault classait sa Griselidis parmi les « nouvelles : c'est-à-dire des récits de choses qui peuvent être arrivées, et qui n'ont rien qui blesse absolument la vraisemblance ». (Préface des Contes en vers, éd. cit., p. 4.)

43. Nouvelles diverses du temps, La Princesse des pretintailles, par Mme la comtesse D. L, mois de novembre, Paris, Ribou, 1702, p. 4.

44. Ibid., p. 37.

45. Lettres nouvelles, de monsieur Boursault. Accompagnées de fables, de remarques, de bons mots, \& d'autres particularitez aussi agréables qu'utiles. Avec sept lettres amoureuses, d'une dame à un cavalier, Paris, $\mathrm{V}^{\mathrm{ve}}$ de T. Girard, 1697. Elles furent de multiples fois rééditées, et dès la seconde édition augmentée. Celle-ci, contenant 13 lettres, parut chez Gosselin en 1699.

46. Journal des Sçavants du lundi 17 mai, p. 218.

47. Le Porte-feuille galant, contenant les entretiens des caffez et autres ouvrages mêlez de prose et de vers (15 juin 1700, Paris, imprimerie de J. Moreau).

48. Sur la production de cette époque, et pour une analyse plus détaillée des contes de Mmes d'Auneuil et Durand, je renvoie à mon article : «1700-1703 : l'éclipse du conte de fées ", L'année 1700, textes réunis par Aurélia Gaillard, Papers on French Seventeenth Century Literature, à paraître. 
49. Ouvr. cité, p. LXV.

50. Contes de Fées et Les Nouveaux Contes des fées, 1698.

51. Euvres meslées, p. 5-6.

52. Ibid., p. 310.

53. Le Recueil de ces Messieurs (1745) est une publication collective de la société du Boutdu-Banc. Un recueil de Caylus, paru la même année et offrant Cinq contes de fées, contient un conte écrit par Mme de Graffigny à partir d'un canevas proposé par Caylus. 54. Pour donner un aperçu de la fortune d'une telle enseigne, énumérons ces titres, sans prétention à l'exhaustivité : Les journées amusantes (Mme de Gomez, 1731, 8 vol. in-12), Amusemens de la campagne, de la cour et de la ville... (1737-1739, 4 vol.), Amusemens agréables... (1738), Amusemens des dames ou Recueil d'histoires galantes des meilleurs auteurs de ce siècle (1711, 1740, 1747, 1753), Amusemens du beau sexe, (1740 à 1774), Amusemens de la campagne ou récréations historiques avec quelques anecdotes secrètes et galantes (1742-1743, 4 vol.), Les Amusemens des fées (1748, 2 vol.), Amusemens spirituels et frivoles (1751), De tout un peu ou les Amusemens de la campagne (1766-1768), Amusemens du jour ou Recueil de petits contes (Mme Mortemart, 1780), et La Bibliothèque de campagne ou Amusemens de l'esprit et du cœur $(1749,176,1785)$ qui avait annoncé 17 volumes, mais en compta bien plus. Le titre Bibliothèque de campagne fut plusieurs fois utilisé. Y furent réédités de nombreux contes de fées, dans des versions souvent remaniées.

55. Le Cabinet des fées, contenant les contes de fées, Amsterdam, 1717 (6 vol.).

56. Les Cabinets des fées, contenant leurs ouvrages en huit volumes, Amsterdam, Michel Charles Le Cene, 1731-1735.

57. Les Cabinets des fées, contenant leurs ouvrages en huit volumes, Amsterdam, M.-M. Rey, 1754-1761, 8 vol. in-12. Un $9^{\mathrm{e}}$ volume paraît en 1735 . Une nouvelle édition, par Mme de $\mathrm{M}^{* * *}$ est publiée en 1754 , chez le même éditeur.

\section{RÉSUMÉS}

Partant des définitions des termes "recueils et recueillir " données par le dictionnaire de l'Académie, l'auteur examine les diverses formes de recueils qui ont été produits avant 1704 . Dans un premier temps, on s'intéresse aux discours théoriques mettant en exergue la collecte d'une matière préexistante et traditionnelle et la transmission orale du conte (démarche exhibée de manière répétitive et militante par $\mathrm{M}^{\mathrm{lle}}$ Lhéritier). Ensuite, quelques implications de l'insertion

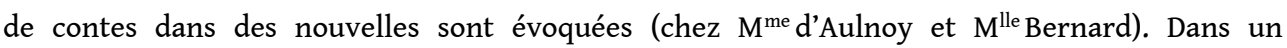
troisième temps, l'accent est mis sur la diversification de la forme du recueil qui s'opère au tournant du siècle, dans certains des ouvrages de $\mathrm{M}^{\text {me }}$ Durand et $\mathrm{M}^{\text {me }} \mathrm{d}$ 'Auneuil, et qui contamine la nature des rares contes merveilleux encastrés. Enfin, on s'intéresse à la dialectique éditoriale conte/recueil. La production de Perrault fournit sur ce point un exemple représentatif et néanmoins assez particulier.

The first collections of fairy tales. Starting from the definitions of «collections " and "to collect " given by the Académie dictionary, A. Defrance examines various forms of collections produced before 1704. She does so in three steps. First the author analyses the theoretical discourses stressing the act of collecting in a traditional manner, and the oral transmission of the 
tale (an approach displayed by Lhéritier in a militant and repetitive way). Then the author briefly considers the insertion of tales in framing short stories. Finally, the author analyses the diversification of the collection itself that changes shape at the turn of the century (Durand and Auneuil) and contaminates the very nature of the few framed fairy tales written at the time. The article concludes on the editorial dialectic tale/collection with Perrault as a representative, but also particular, example.

\section{AUTEUR}

\section{ANNE DEFRANCE}

Université Bordeaux 3 - Umr Lire 